\title{
On the Stability of the Orbit and the Invariant Set of Thomson's Vortex Polygon in a Two-Fluid Plasma
}

\author{
L. G. Kurakin, I. A. Lysenko
}

The motion of the system of $N$ point vortices with identical intensity $\Gamma$ in the Alfven model of a two-fluid plasma is considered. The stability of the stationary rotation of $N$ identical vortices disposed uniformly on a circle with radius $R$ is studied for $N=2, \ldots, 5$. This problem has three parameters: the discrete parameter $N$ and two continuous parameters $R$ and $c$, where $c>0$ is the value characterizing the plasma. Two different definitions of the stability are used - the orbital stability and the stability of a three-dimensional invariant set founded by the orbits of a continuous family of stationary rotations. Instability is interpreted as instability of equilibrium of the reduced system. An analytical analysis of eigenvalues of the linearization matrix and the quadratic part of the Hamiltonian is given. As a result, the parameter space $(N, R, c)$ of this problem for two stability definitions considered is divided into three parts: the domain $\boldsymbol{A}$ of stability in an exact nonlinear problem setting, the linear stability domain $\boldsymbol{B}$, where the nonlinear analysis is needed, and the domain of exponential instability $\boldsymbol{C}$. The application of the stability theory of invariant sets for the systems with a few integrals for $N=2,3,4$ allows one to obtain new statements about the stability in the domains, where nonlinear analysis is needed in investigating the orbital stability.

Keywords: point vortex, two-fluid plasma, stability, stationary rotation, Hamiltonian system, invariant set

Received June 26, 2019

Accepted October 14, 2019

Research was financially supported by Southern Federal University, 2020 (Ministry of Science and Higher Education of the Russian Federation), VnGr/2020-04-IM.

Leonid G. Kurakin

kurakin@math.rsu.ru

Southern Mathematical Institute, Vladikavkaz Scientific Center of RAS

ul. Markusa 22, Vladikavkaz, 362027 Russia

Water Problems Institute of RAS

ul. Gubkina 3, Moscow, 119333 Russia

Irina A. Lysenko

irlys@sfedu.ru

Southern Federal University

ul. Milchakova 8a, Rostov-on-Don, 344090 Russia 


\section{Introduction}

Systems of point vortices located uniformly on a circle (Thomson's vortex $N$-gon) have been studied for a long time from different points of view by Kelvin (W. Thomson) [7, 23], J. J. Thomson [24] and T. H. Havelock [5], who investigated this problem completely in the linear setting for the Kirchhoff model. The review of experimental and theoretical works on the solution of this problem in an exact nonlinear setting is given in the paper [9].

The stability of the Thomson's $N$-gon for geostrophic (Bessel) vortices was studied in [14, 18, $21,22]$. In the paper [14] the stability problem of a vortex polygon becomes that of investigating the stability of the equilibrium family of a Hamiltonian system with a cyclic variable, and the Routh theory is used for such systems [19].

This problem for a two-layer rotating fluid for arbitrary quantities of vortices $N$ has been studied in [15].

This paper is concerned with the investigation of the stability problem for Thomson's vortex $N$-gon in the model of a two-fluid plasma for a number of vortices $2 \leqslant N \leqslant 5$. The analysis is based on the results obtained in $[15,16]$.

The stability problem of Thomson's vortex $N$-gon in the Alfven model of a two-fluid plasma was considered in particular cases in [3]. This model was proposed by Swedish scientist Alfven in the framework of the theory of magnetic hydrodynamics [1]. It is based on Kelvin's achievements in the study of vortices and follows the Maxwell electromagnetic theory. Somewhat later, Batchelor drew an analogy between vorticity and a magnetic field [2]. The equations of two-fluid hydrodynamics describe electrons and ions as two conducting fluids connected with each other by the friction force and electromagnetic fields [8].

The complex potentials of the currents induced by a vortex filament of intensity æ, which is located at point $z_{0}$, for the models of a two-fluid plasma [3] and a two-layer fluid [20] have the form

$$
w(z)=i æ\left[\ln \left(z-z_{0}\right) \pm c K_{0}\left(\left|z-z_{0}\right|\right)\right], \quad c>0,
$$

where the signs "+" and "-" correspond to the model of a two-fluid plasma and the model of a two-layer fluid, respectively. Here, $K_{0}$ is a modified Bessel function of zero order. For the two-fluid plasma the parameter $c$ doesn't make a special denomination, and its physical meaning is presented in [3].

In the limiting case $c=0$ both models are transformed into Kirchhoff's model. As $c \rightarrow+\infty$ $(c \rightarrow-\infty)$ the model of a two-fluid plasma (two-layer fluid) does not turn into the model of Bessel vortices. However, the results for the stability problem of Thomson's vortex $N$-gon for these models for $c \rightarrow \pm \infty$ and for the model of Bessel vortices coincide.

The eigenvalues of the corresponding linearization matrix for the stability problem of Thomson's vortex $N$-gon in a two-fluid plasma were numerically investigated in [3]. The results are presented as graphs [3, Fig. 9] for the specific values of the problem parameters: $N=4, \ldots, 10$ on the interval $0<R \leqslant 8$ for $c=4$, suggesting that these conclusions are typical for all values of the parameter $c$. The values of radius $R$ for which the exponential instability takes place are found. For the remaining values of the parameter $R$ there is stability in the linear problem setting, but the question about the stability in an exact nonlinear setting remains open.

A partial answer to it is obtained in this paper, where the problem is solved analytically for the model of a two-fluid plasma at all possible values of the parameter $c \geqslant 0$ and $0<R \leqslant 10$ for the cases of $N=2, \ldots, 5$. Here, an analysis of the eigenvalues of the linearization matrix and the quadratic part of the Hamiltonian is presented. We use some statements about the stability of Thomson's vortex $N$-gon obtained in [15, Section 3] for systems of interacting particles, 
which are defined by the Hamiltonian of a general form depending only on the mutual distances between the particles:

$$
\mathcal{H}(\mathbf{q}, \mathbf{p})=-\frac{\Gamma}{4 \pi} \sum_{1 \leqslant j<k \leqslant N} W\left(\sqrt{\left(q_{j}-q_{k}\right)^{2}+\left(p_{j}-p_{k}\right)^{2}}\right) .
$$

Here, $W$ is an arbitrary smooth function defined on the set $\mathbb{R}_{+} ; \mathbf{q}=\left(q_{1}, \ldots, q_{N}\right)$ and $\mathbf{p}=\left(p_{1}, \ldots, p_{N}\right)$ are Cartesian coordinates of the particles; and $\Gamma \in \mathbb{R}$ is some parameter. As a result, the space of parameters $(N, R, c)$ is divided into three parts corresponding to the possible cases $\boldsymbol{A}, \boldsymbol{B}$, and $\boldsymbol{C}$, depending on the type of stability or its absence (see Theorem 1 below). The analytical results obtained are consistent with the numerical results of the paper [3] and generalize them.

Conclusions about the orbital stability for $N=3,4,5$ were published in [16]. The stability theory of invariant sets for systems with a few integrals [6] is applied for the study of another type of stability - the stability of a three-dimensional invariant set formed by the orbits of a continuous family of stationary rotations. This approach allows one to obtain new statements about the stability in domains of type $\boldsymbol{B}$, which were obtained in investigating the orbital stability.

\section{Formulation of the Problem}

The system of $N$ identical point vortices with intensity $\Gamma$ in the Alfven two-fluid plasma is considered. Their motion is described by Hamiltonian $\mathcal{H}(\mathbf{q}, \mathbf{p})$ given by the formula (1.2), where the function $W[3]$ is

$$
W(\xi)=\ln (\xi)+c K_{0}(\xi), \quad c \geqslant 0 .
$$

In the complex variables $z_{k}=q_{k}+i p_{k}, \bar{z}_{k}=q_{k}-i p_{k}$ we obtain a system with the Hamiltonian $H(\mathbf{z}, \overline{\mathbf{z}})=\mathcal{H}(\mathbf{q}(\mathbf{z}, \overline{\mathbf{z}}), \mathbf{p}(\mathbf{z}, \overline{\mathbf{z}}))$, where $\mathbf{z}=\left(z_{1}, \ldots, z_{N}\right), \overline{\mathbf{z}}=\left(\bar{z}_{1}, \ldots, \bar{z}_{N}\right):$

$$
\dot{\bar{z}}_{k}=2 i H_{z_{k}}, \quad \dot{z}_{k}=-2 i H_{\bar{z}_{k}}, \quad k=1, \ldots, N .
$$

The analytical study is conducted using the parameter $c$, and the results in the form of graphs are presented for clarity for the parameter $\alpha$ :

$$
\alpha=\frac{1-c}{1+c}, \quad-1<\alpha \leqslant 1
$$

The following relation is valid:

$$
c=\frac{1-\alpha}{1+\alpha}, \quad c \geqslant 0
$$

The system (2.2) has an exact solution

$$
z_{k}=e^{i \omega_{N} t} u_{k}, \quad u_{k}=R e^{2 \pi i(k-1) / N}, \quad R>0, \quad k=1, \ldots, N,
$$

corresponding to the stationary rotation of the system of $N$ point vortices located at the vertices of the regular $N$-gon. 
The angular velocity $\omega_{N}$ is given by the expression

$$
\begin{gathered}
\omega_{N}(R, c)=\frac{\Gamma}{8 \pi R} \sum_{m=1}^{N-1} W^{\prime}\left(R B_{m}\right) B_{m}, \quad \text { where } \\
W^{\prime}\left(R B_{m}\right)=\frac{1}{R B_{m}}-c K_{1}\left(R B_{m}\right), \quad B_{m}=\sqrt{2-2 C_{m}}, \quad C_{m}=\cos \frac{2 m \pi}{N} .
\end{gathered}
$$

The function $\omega_{N}(R, c)$ increases by variable $c$ and monotonously decreases by variable $R$. The direction of rotation of the system of vortices for each $N$ is changed during the transition of parameter $c$ across the curve

$$
c_{N}(R)=\frac{N-1}{R \sum_{m=1}^{N-1} K_{1}\left(R B_{m}\right) B_{m}},
$$

on which the angular velocity is $\omega_{N}(R, c)=0$. In the variables $(R, \alpha)$ the curves $\alpha_{N}(R)=$ $=\frac{1-c_{N}(R)}{1+c_{N}(R)}$ are presented in Fig. 1 . The functions $\alpha_{N}(R)$ monotonously decrease by variable $R$ and increase by $N$.

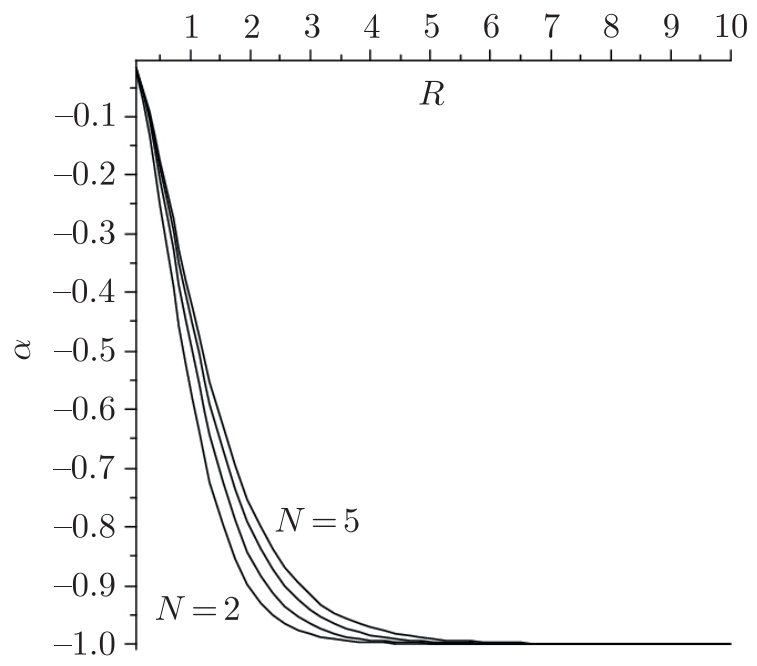

Fig. 1. The curves $\alpha_{N}(R)$ corresponding to the condition $\omega_{N}=0, N=2, \ldots, 5$. The graphs are arranged from top to bottom in the order of decreasing parameter $N$.

Further, the stability of rotation (2.5), (2.6) of the regular vortices $N$-gon for the model with the Hamiltonian (1.2), (2.1) is studied.

\section{Orbital stability of Thomson's vortex polygon in a two-fluid plasma}

The problem of orbital stability for this model of a two-fluid plasma for $N=3,4,5$ was considered in [16]. 
Let the values $\lambda_{j k}(j=0,1,2 ; k=1, \ldots, N)$ be given by the formulas

$$
\begin{aligned}
\lambda_{1 k} & =-\frac{c}{8 R^{2}} \sum_{m=1}^{N-1}\left(\left(1+\cos \frac{2 \pi m k}{N}\right) B_{m}^{2} K_{0 m}+4\left(1-2 C_{m}+\cos \frac{2 \pi m k}{N}\right) \frac{K_{1 m}}{R B_{m}}\right)+ \\
& +\frac{2(N-1)-k(N-k)}{4 R^{4}}, \quad K_{0 m}=K_{0}\left(R B_{m}\right), K_{1 m}=K_{1}\left(R B_{m}\right), \\
\lambda_{2 k} & =-\frac{c}{2} R^{2} \sum_{m=1}^{N-1}\left(1-\cos \frac{2 \pi m k}{N}\right)\left(\frac{K_{1 m}}{R B_{m}}+\frac{1}{2}\left(1+C_{m}\right) K_{0 m}\right)+\frac{k(N-k)}{4}, \\
\lambda_{0 k} & =-\frac{c}{4} \sum_{m=1}^{N-1} S_{m} K_{0 m} \sin \frac{2 \pi m k}{N}, \quad \text { where } \quad S_{m}=\sin \frac{2 m \pi}{N},
\end{aligned}
$$

and polynomials $P(N, k, \Lambda)$ :

$$
P(N, k, \Lambda)=\Lambda^{2}+p_{1}(N, k) \Lambda+p_{0}(N, k), \quad k=1, \ldots, N,
$$

where the coefficients are

$$
p_{1}(N, k)=-\left(\lambda_{1 k}+\lambda_{2 k}\right), \quad p_{0}(N, k)=\lambda_{1 k} \lambda_{2 k}-\lambda_{0 k}^{2} .
$$

The eigenvalues of the corresponding linearization matrix of the problem are calculated by the formula

$$
\sigma_{k}^{ \pm}=-2 i \lambda_{0 k} \pm 2 \sqrt{-\lambda_{1 k} \lambda_{2 k}}, \quad k=1, \ldots, N .
$$

The following theorem [16] for the stationary rotation (2.5), (2.6) of the model with the Hamiltonian (1.2), (2.1) is valid.

Theorem 1. The parameter space $(N, R, c)$ is divided into three nonoverlapping domains corresponding to three possible cases $\boldsymbol{A}, \boldsymbol{B}, \boldsymbol{C}$ :

Case $\boldsymbol{A}$. The stationary rotation (2.5), (2.6) is orbitally stable in an exact nonlinear problem setting if the coefficients $p_{0}(N, k)$ of the polynomials $P(N, k, \Lambda)$ given by equalities $(3.4),(3.5)$ are positive for all $k=1, \ldots,\left\lfloor\frac{N}{2}\right\rfloor$ :

$$
p_{0}(N, k)=\lambda_{1 k}(N, R, c) \lambda_{2 k}(N, R, c)-\lambda_{0 k}^{2}(N, R, c)>0, \quad k=1, \ldots,\left\lfloor\frac{N}{2}\right\rfloor,
$$

and all polynomials $P(N, k, \Lambda)$ have the roots of identical sign for each $k=1, \ldots,\left\lfloor\frac{N}{2}\right\rfloor$, where $\left\lfloor\frac{N}{2}\right\rfloor$ is the integer part of the number $\frac{N}{2}$.

Case $\boldsymbol{C}$. The instability in this problem takes place if at least one eigenvalue (3.6) of the linearization matrix $\mathbf{L}$ has a positive real part. It means that the inequality

$$
\lambda_{1 k}(N, R, c) \lambda_{2 k}(N, R, c)<0
$$

is valid for at least one $k=2, \ldots,\left\lfloor\frac{N}{2}\right\rfloor$.

Case $\boldsymbol{B}$. If the conditions of cases $\boldsymbol{A}$ and $\boldsymbol{C}$ are disrupted, then in the problem of stability of stationary rotation (2.5), (2.6) nonlinear analysis is required. The linear stability takes place. 
Further, the conclusions about the orbital stability obtained by applying Theorem 1 for $N=2, \ldots, 5$ are presented.

In Figs. 2, 3 the domains corresponding to all possible cases $\boldsymbol{A}, \boldsymbol{B}$ and $\boldsymbol{C}$ are designated, respectively, by light color, dark color and by hatching.

The domains of these different types are separated by the curves $l_{k i}^{(N)}=l_{k i}^{(N)}(R)$ and $s_{k i}^{(N)}=s_{k i}^{(N)}(R)$ (where $k=1, \ldots,\left\lfloor\frac{N}{2}\right\rfloor$ is the number of polynomial, $i=1,2$ is the number of solution), on which the left-hand sides of inequalities (3.8) and (3.7) vanish, respectively.

All the shown curves are defined by the analytical formulas presented in [16].

For each $N$ considered the equality $\omega_{N}(R)=0$ holds on the curves $s_{11}^{(N)}(R)$.

On all sections of the presented curves, located outside the instability domain $C$ and on its border, the conditions corresponding to case $\boldsymbol{B}$ are valid.

The case $N=2$. The case $\boldsymbol{A}$ of stability in an exact nonlinear problem setting takes place for all parameter values except at the points of the curve presented in Fig. 1 for $N=2$. On this curve corresponding to zero angular velocity $\omega_{N}=0$ the condition of case $\boldsymbol{B}$ is valid, and the problem requires nonlinear analysis.

The case $N=3$. The stability diagram of the stationary rotation (2.5), (2.6) is shown in Fig. 2a.

The case $N=4$. The stability diagram for $N=4$ is displayed in Fig. 2b. Here, $O_{4}(3.122 ;-0.951)$ is the point of intersection of the curves $s_{11}^{(4)}$ and $s_{21}^{(4)}$. Note that for $N=4$ the lines $l_{2 i}^{(4)}$ and $s_{2 i}^{(4)}$ for each $i=1,2$ coincide.

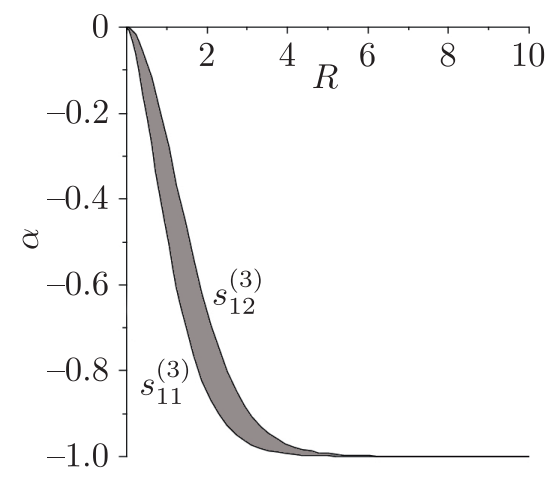

(a)

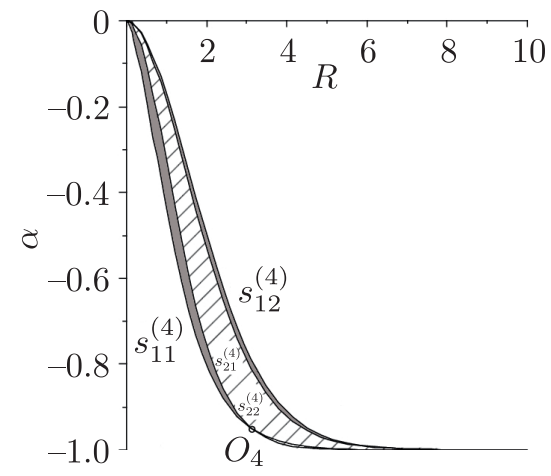

(b)

Fig. 2. Diagrams of orbital stability: (a) for $N=3$; (b) for $N=4$. In the light domain the stability in an exact nonlinear problem setting takes place (the case $\boldsymbol{A}$ ); in the dark domain the problem requires nonlinear analysis (case $\boldsymbol{B}$ ); in the hatched domain the rotation of $N$ vortices is unstable (case $\boldsymbol{C}$ ).

The case $N=5$. The stability diagram is presented in Fig. 3 for the parameter values $0<R \leqslant 10$. The coordinates of marked points are $O_{5}(1.724 ;-0.689)$ and $D_{5}(3.76 ;-1)$, where $s_{11}^{(5)}=s_{21}^{(5)}$ in $O_{5}$, and $s_{21}^{(5)}=-1$ at the point $D_{5}$. 


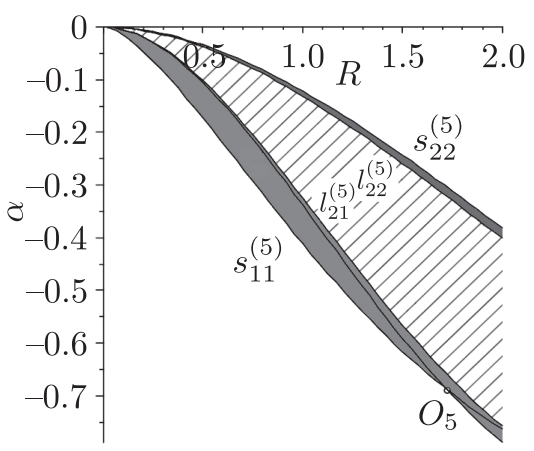

(a)

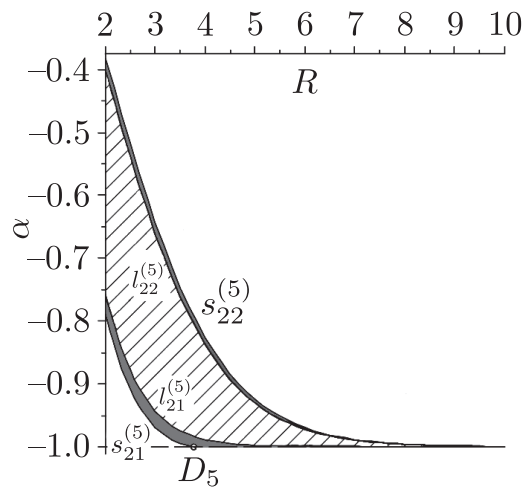

(b)

Fig. 3. Diagrams of orbital stability for $N=5$ : (a) $0<R \leqslant 2$; (b) $2 \leqslant R \leqslant 10$. The domains designations are the same as in Fig. 2.

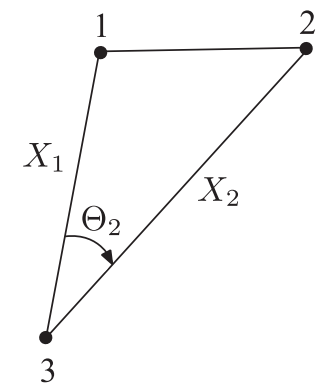

(a)

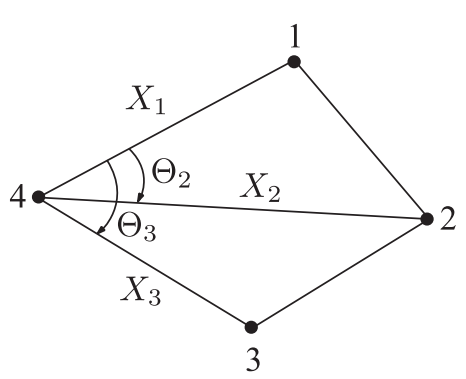

(b)

Fig. 4. Designations of the variables: (a) for a triangle; (b) for a tetragon.

\section{Stability of an invariant set of Thomson's vortex polygon in a two-fluid plasma}

The system of equations (2.2) is invariant under translation. Therefore, the existence of the solution (2.5) implies the existence of a family of stationary rotations

$$
z_{k}(t)=u_{k} e^{i \omega_{N} t}+\eta, \quad k=1, \ldots, N, \quad \eta \in \mathbb{C} .
$$

The stability of the three-dimensional invariant set $\mathcal{M}_{N}$ formed by the orbits of this continuous family (4.1) is studied.

Let the vortices for each $N$ be numbered clockwise and $X_{i}(i=1, \ldots, N-1)$ are the distances from the $N$ th vortex to all other vortices with number $i$. Also, we denote the angles between these segments as follows: $\widehat{X}_{i}, X_{j}=\Theta_{j}-\Theta_{i}$, where $1 \leqslant i<j \leqslant N-1$ and $\Theta_{1}=0$.

So, we need to use only $2 N-3$ variables in order to express the distances between all $N$ vortices. The coordinates of the vector $\mathbf{U}=\left(X_{1}, \ldots, X_{N-1}, \Theta_{2}, \ldots, \Theta_{N-1}\right)$ are these variables. For each $N$ the point of the space $\mathbb{R}^{2 N-3}$ with coordinates of vector $\mathbf{U}_{*}$ corresponds to the invariant set $\mathcal{M}_{N}$, where

$$
\begin{array}{ll}
\text { for } \quad N=2: & \mathbf{U}_{*}=2 R ; \quad \text { for } \quad N=3: \quad \mathbf{U}_{*}=\left(\sqrt{3} R, \sqrt{3} R, \frac{\pi}{3}\right) ; \\
\text { for } \quad N=4: & \mathbf{U}_{*}=\left(\sqrt{2} R, 2 R, \sqrt{2} R, \frac{\pi}{4}, \frac{\pi}{2}\right) .
\end{array}
$$


The methods of the stability theory for invariant sets in systems with a few integrals [6] are used. The first integrals $V(\mathbf{U})$ and $L(\mathbf{U})$ of the initial system $(2.2)$ with Hamiltonian $H$ are considered (see, for example, $[4,13])$ :

$$
\begin{gathered}
L(\mathbf{U})=\sum_{k=1}^{N-1} X_{k}^{2}+\sum_{1 \leqslant i<j \leqslant N-1}\left(X_{i}^{2}+X_{j}^{2}-2 X_{i} X_{j} \cos \left(\Theta_{j}-\Theta_{i}\right)\right), \\
V(\mathbf{U})=-\sum_{k=1}^{N-1} W\left(X_{k}\right)-\sum_{1 \leqslant i<j \leqslant N-1} W\left(\sqrt{X_{i}^{2}+X_{j}^{2}-2 X_{i} X_{j} \cos \left(\Theta_{j}-\Theta_{i}\right)}\right) .
\end{gathered}
$$

The invariant set $\mathcal{M}_{N}$ is stable if the function $I$

$$
I(\mathbf{U})=\left(V(\mathbf{U})-V\left(\mathbf{U}_{*}\right)\right)^{2}+\left(L(\mathbf{U})-L\left(\mathbf{U}_{*}\right)\right)^{2}
$$

has a local minimum at the point with coordinates of the vector $\mathbf{U}_{*}$.

The analysis presented above yields the following conclusions on the stability of the invariant set $\mathcal{M}_{N}$ for $N=2,3,4$.

The case $N=2$. The stability of the invariant set $\mathcal{M}_{2}$ in an exact nonlinear problem setting takes place for all parameter values.

The case $N=3$. The stability of the invariant set $\mathcal{M}_{3}$ in an exact nonlinear problem setting takes place everywhere on the parameter plane $(R, \alpha)$ except at the points of the curve $s_{12}^{(3)}$ presented in Fig. 2a.

The case $N=4$. The stability of the invariant set $\mathcal{M}_{4}$ in an exact nonlinear problem setting takes place in the domains corresponding to the case $\boldsymbol{A}$ of orbital stability (white domains in Fig. 2b). Apart from this, the invariant set $\mathcal{M}_{4}$ is stable in the domain $G$, which is shown in Fig. $2 \mathrm{~b}$ between the curves $s_{11}^{(4)}$ and $s_{21}^{(4)}$ from the point $(0,0)$ to their intersection at the point $\mathrm{O}_{4}$, and is colored gray.

\section{References}

[1] Alfvén, H., On the Existence of Electromagnetic-Hydromagnetic Waves, Arc. f. Mat. Ast. Fys., 1942, vol. 29B, no. 2, 7 pp.

[2] Batchelor, G. K., On the Spontaneous Magnetic Field in a Conducting Liquid in Turbulent Motion, Proc. Roy. Soc. London Ser. A, 1950, vol. 201, no. 1066, pp. 405-416.

[3] Bergmans, J., Kuvshinov, B. N., Lakhin, V. P., and Schep, T. J., Spectral Stability of Alfvén Filament Configurations, Phys. Plasmas, 2000, vol. 7, no. 6, pp. 2388-2403.

[4] Borisov, A. V. and Mamaev, I.S., Mathematical Methods in the Dynamics of Vortex Structures, Izhevsk: R\&C Dynamics, Institute of Computer Science, 2005 (Russian).

[5] Havelock, T.H., The Stability of Motion of Rectilinear Vortices in Ring Formation, Philos. Mag., 1931, vol. 11, no. 70, pp. 617-633.

[6] Karapetyan, A. V., Invariant Sets of Mechanical Systems: Lyapunov's Methods in Stability and Control, Math. Comput. Modelling, 2002, vol. 36, no. 6, pp.643-661.

[7] Kelvin, W. T., Mathematical and Physical Papers: Vol. 4, Cambridge: Cambridge Univ. Press, 1910, pp. $162-164$.

[8] Krall, N. A. and Trivelpiece, A. W., Principles of Plasma Physics, New York: McGraw-Hill, 1973. 
[9] Kurakin, L. G. and Yudovich, V. I., The Stability of Stationary Rotation of a Regular Vortex Polygon, Chaos, 2002, vol. 12, no. 3, pp. 574-595.

[10] Kurakin, L. G. and Yudovich, V. I., On the Nonlinear Stability of the Steady Rotation of a Regular Vortex Polygon, Dokl. Phys., 2002, vol.47, no.6, pp.465-470; see also: Dokl. Akad. Nauk, 2002, vol. 384, no. 4, pp. 476-482.

[11] Kurakin, L. G., Stability, Resonances, and Instability of the Regular Vortex Polygons in the Circular Domain, Dokl. Phys., 2004, vol. 49, no.11, pp.658-661; see also: Dokl. Akad. Nauk, 2004, vol. 399, no. 1 , pp. $52-55$.

[12] Kurakin, L. G. and Ostrovskaya, I. V., Nonlinear Stability Analysis of a Regular Vortex Pentagon Outside a Circle, Regul. Chaotic Dyn., 2012, vol.17, no. 5, pp. 385-396.

[13] Kurakin, L. G., Ostrovskaya, I. V., and Sokolovskiy, M.A., On the Stability of Discrete Tripole, Quadrupole, Thomson' Vortex Triangle and Square in a Two-Layer/Homogeneous Rotating Fluid, Regul. Chaotic Dyn., 2016, vol. 21, no. 3, pp. 291-334.

[14] Kurakin, L. G. and Ostrovskaya, I. V., On Stability of the Thomson's Vortex $N$-Gon in the Geostrophic Model of the Point Bessel Vortices, Regul. Chaotic Dyn., 2017, vol.22, no.7, pp. 865-879.

[15] Kurakin, L. G., Lysenko, I. A., Ostrovskaya, I. V., and Sokolovskiy, M. A., On Stability of the Thomson's Vortex $\mathrm{N}$-Gon in the Geostrophic Model of the Point Vortices in Two-Layer Fluid, J. Nonlinear Sci., 2019, vol. 29, no. 4, pp. 1659-1700.

[16] Lysenko, I. A., On Stability of a Vortex Triangle, Square and Pentagon in the Two-Fluid Plasma, Izv. Vyssh. Uchebn. Zaved. Severo-Kavkazskii Region. Natural Science, 2019, no.1, pp. 17-23 (Russian).

[17] Markeev, A. P., Libration Points in Celestial Mechanics and Space Dynamics, Moscow: Nauka, 1978 (Russian).

[18] Morikawa, G. K. and Swenson, E. V., Interacting Motion of Rectilinear Geostrophic Vortices, Phys. Fluids, 1971, vol. 14, no. 6, pp. 1058-1073.

[19] Routh, E. J., A Treatise on the Stability of a Given State of Motion, Particularly Steady Motion, London: Macmillan, 1877.

[20] Sokolovskiy, M. A. and Verron, J., Dynamics of Vortex Structures in a Stratified Rotating Fluid, Atmos. Oceanogr. Sci. Libr., vol. 47, Cham: Springer, 2014.

[21] Stewart, H. J., Periodic Properties of the Semi-Permanent Atmospheric Pressure Systems, Quart. Appl. Math., 1943, vol. 1, pp. 262-267.

[22] Stewart, H. J., Hydrodynamic Problems Arising from the Investigation of the Transverse Circulation in the Atmosphere, Bull. Amer. Math. Soc., 1945, vol. 51, pp. 781-799.

[23] Thomson, W., Floating Magnets (Illustrating Vortex Systems), Nature, 1878, vol. 18, pp. 13-14.

[24] Thomson, J. J., Treatise on the Motion of Vortex Rings, London: Macmillan, 1883, pp. 94-108. 